\title{
Pancreatic lipoma and its differentiation from various fat containing lesions in the pancreas: an imaging guide
}

\begin{abstract}
Lipoma of the pancreas is a rare benign tumor which is usually discovered incidentally on imaging. Being innocuous in nature it does not require surgical removal and therefore needs to be differentiated from various pancreatic masses. Unlike other pancreatic tumors, it can be confirmed on CT or MRI imaging and does not require invasive histopathological examination to establish a definite diagnosis. We present a case of pancreatic lipoma in a 46year old female, detected incidentally on ultrasound and confirmed on Computed Tomography by demonstrating the characteristic imaging features of lipoma and thus, no further histopathological confirmation was required. This case report presents a brief review of literature re-emphasizing the importance of imaging in distinguishing lipoma from other fat containing lesions of the pancreas and thus re-enforcing the "masterly inactivity" policy for these lesions. A confident imaging diagnosis of a pancreatic mass being a lipoma would obviate the anxiety of the patient as well as the clinician regarding the need for further management.
\end{abstract}

Volume 4 Issue 5 - 2017

\section{Mohammad Ahmad, Shuchi Bhatt, Anupama Tandon, Samrat Mandal, Daizy Garg \\ Department of Radio-Diagnosis, University College of Medical} Sciences, India

\section{Correspondence: Shuchi Bhatt, Department of Radio-}

Diagnosis, University College of Medical Sciences (University of Delhi), Guru Teg Bahadur Hospital, Dilshad Garden , New Delhi95, India, Tel 9 | I | 22586262, 244 I, 240 I, Fax 009 | | | 2259049, Emaildrshuchi@hotmail.com

Received: October 27, 2017 | Published: November 24, 2017

Keywords: lipoma, liposarcoma, pancreas, computed tomography

\section{Introduction}

Pancreatic tumors originate from mesenchymal or epithelial cells, or from non-ductal structures. Epithelial tumors constitute the largest group $(85 \%)$ including adenocarcinoma, non-ductal tumors for $5 \%$ $15 \%$, and mesenchymal for $1 \%,{ }^{1}$ with those containing fat being the rarest. Lipomas show characteristic imaging features, identification of which allow a correct diagnosis without any histopathological confirmation. $^{2}$

\section{Case report}

A middle-aged woman presented with upper abdominal pain for 3months. Clinical examination and routine laboratory investigations were normal. Ultrasound (USG) revealed a well-defined, irregular shaped, solid, hypoechoic lesion (Figure 1) in head of pancreas, which was otherwise normal in size and echogenicity. Pancreatic duct, biliary system, and other solid organs were normal. Evaluation with contrast enhanced CT (CECT) demonstrated a well- defined, intrapancreatic lesion (Figure 2) of size $1.9 \mathrm{~cm} \times 1 \mathrm{~cm}$ with a uniform fat density ( -143 to $-150 \mathrm{HU})$ and no contrast enhancement. Characteristic CT findings confirmed a pancreatic lipoma.

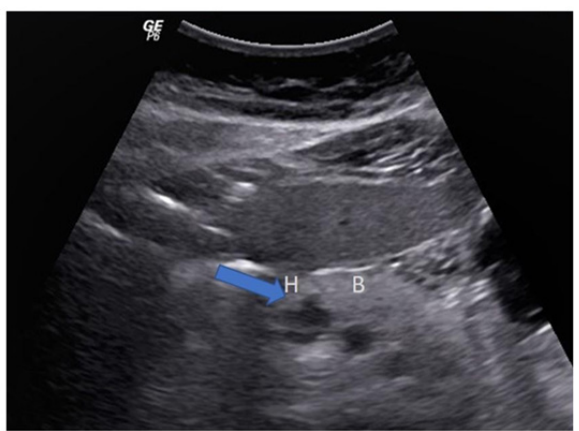

Figure I Abdominal US showing a well defined, hypoechoic lesion with a lobulated contour (blue arrow) in pancreatic head. No ductal dilatation is apparent.

$H$, head of pancreas; $B$, body of pancreas.

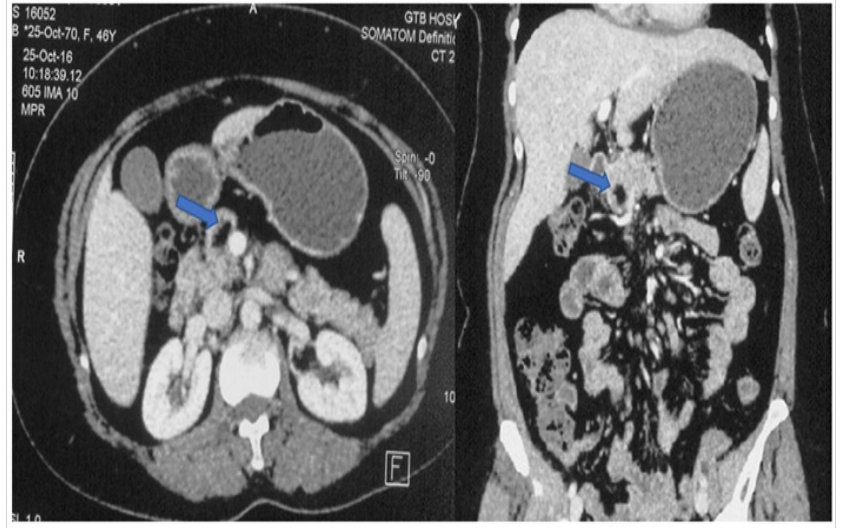

Figure 2 Axial and coronal reformatted images of contrast enhanced CT showing a small, well circumscribed hypodense focal lesion (blue arrow) in pancreatic head, no central or peripheral enhancement present.

\section{Discussion}

The most frequent pancreatic tumor is adenocarcinoma (85\%), followed by non-ductal carcinoma (5-15\%). Mesenchymal tumours account for $1 \%$ only, and they may be benign (lymphangioma, lipoma, fibroma, neurofibroma, schwannoma, haemangioma, haemangioendothelioma, leiomyoma and desmoid tumours) or malignant (lymphoma, pancreatoblastoma, liposarcoma, fibrous histiocytoma, haemangiopericytoma and neuroectodermal neoplasms) which is identified on histopathology. ${ }^{1}$

Histologically, lipoma is an encapsulated mass of mature adipose cells arranged in lobules, and may contain fine connective tissue septa inside. A thin capsule differentiates a lipoma from lipomatosis and facilitates its enucleation. It may occur anywhere in the body, and frequently in the gastrointestinal tract when intra-abdominal, and rarely within the pancreas. The incidence of pancreatic lipoma was calculated to be $0.083 \%,{ }^{3}$ but recently, a retrospective review of 100 abdominal CT revealed much higher incidence. ${ }^{4}$ This suggests 
cases are not being reported or missed rather than being rare. Being an innocuous lesion, many radiologists may not feel the need to mention it. Moreover, small lesions may be difficult to recognize on thick slices due to volume averaging effects. It is usually less than $5 \mathrm{~cm}$ and clinically silent or may present with abdominal pain, biliary or pancreatic duct obstruction when large. Symptomatic intra-pancreatic lipoma may require surgical treatment. On USG, pancreatic lipoma may appear hypo-, iso- or hyperechoic. It is a small hypoechoic lesion with sharp irregular borders ${ }^{3,5}$ as was also present in our case. Legmann et al. ${ }^{5}$ identified characteristic features of homogenous fat density (HU -80 to -120 ) with no central or peripheral enhancement on CT. A sharp demarcation with no evidence of infiltration into surrounding pancreatic or extra-pancreatic tissues shows its benign nature. Histologic confirmation is not required as a definite diagnosis can be made on the basis of CT alone. Lipomas appear hyper intense on both $\mathrm{T} 1$ and $\mathrm{T} 2$ weighted sequences, similar to intra-abdominal and subcutaneous fat. Fat-suppressed images show homogeneous suppression of signal intensity within the tumour. Like CT, MRI also does not show contrast enhancement. Butler et al. ${ }^{6}$ followed intra-pancreatic lipomas for 12years; majority of which were asymptomatic at presentation, none required intervention, or showed interval growth or change in imaging appearance. This proved that lipomas are stable in size and morphology. Interval followup is required to prove their benign nature to differentiate them from early liposarcoma. Only one case report exists in literature showing a morphological change and increase in size in a pancreatic lipoma sevenyears after the diagnosis was established. ${ }^{7}$ A liposarcoma was proved on endoscopic ultrasound guided FNAC from the lesion. Other authors have also highlighted the advantages of imaging guidance using endoscopic ultrasound to obtain better tissue yield from the suspicious lesion in the pancreas and also to prevent tumor seedling and formation of a pancreatic fistula.

Important differential diagnoses considered include fatty replacement, pseudohypertrophic lipomatosis, teratoma and liposarcoma. Differentiation between pancreatic lipoma and focal fatty infiltration of the pancreas is difficult as both have a density (CT) and signal (MR) typical of fatty tissue, but the latter is a heterogeneous lesion with poorly defined margins and faint, nonhomogenous contrast enhancement. ${ }^{9}$ Although this differentiation is not required, as neither needs to be treated medically or surgically. Detecting presence of fatty tissue, rules out the diagnosis of adenocarcinoma and pancreatic neuroendocrine tumour. Pseudohypertrophic lipomatosis is a rare condition showing pancreatic enlargement due to increase in fat content subsequent to decreased parenchymal cells, especially exocrine tissue with intact islets of Langerhans. MRI is very useful and shows almost total replacement of pancreatic parenchyma by fatty tissue. Teratoma is also rare in the pancreas and is diagnosed when a variegated density lesion is present or both calcification and fat are present in the lesion. It is generally asymptomatic but requires surgical treatment. Liposarcoma are slowly growing malignant fatty tumor, most commonly seen in extremities and retroperitoneum and rarely in the pancreas. A large size, inhomogeneous attenuation due to soft- tissue within the fatty mass and poor definition favors a liposarcoma.
An incidental pancreatic lipoma can be managed with masterly inactivity. Monitoring with serial imaging will detect lesions causing obstructive symptoms or development of malignancy within it. Surgery is offered in such cases, unlike in an intra-pancreatic lipoma. Histopathological confirmation of a malignant change in intra-pancreatic lipoma is essential before its removal.

\section{Conclusion}

Imaging plays an important role in diagnosing and differentiating intra-pancreatic lipoma, an innocuous lesion from other fat containing lesions including the more sinister liposarcoma. The typical presence of homogenous fat (negative HU values on CT or hyperintensity on both $\mathrm{T} 1$ and $\mathrm{T} 2$ weighted images on MRI) in a well-defined intra-pancreatic lesion having no internal or peripheral contrast enhancement and no evidence of surrounding tissue infiltration confirms a lipoma. When follow-up imaging shows the possibility of the lipoma turning malignant, it can be safely subjected to an endoscopic ultrasound guided FNAC to confirm a liposarcoma before the surgical excision is undertaken. Thus, a radiologist plays a crucial role in exercising masterly inactivity for a pancreatic lipoma, only to suggest an intervention at the appropriate time.

\section{Acknowledgements}

None.

\section{Conflict of interest}

Author declares that there is no conflict of interest.

\section{References}

1. Raut CP, Fernandez-del Castillo C. Giant lipoma of the pancreas: case report and review of lipomatous lesions of the pancreas. Pancreas. 2003;26(1):97-99.

2. Stadnik A, Cieszanowsk A, Bakon L, et al. Pancreatic lipoma: An incidentaloma which can resemble cancer-analysis of 13 cases studied with CT and MRI. Pol J Radiol. 2012;77(3):9-13.

3. Hois EL, Hibbeln JF, Sclamberg JS. CT appearance of incidental pancreatic lipomas: a case series. Abdom Imaging. 2006;31(3):332-338.

4. Gossner J. Pancreatic lipomas - prevalence in patients undergoing abdominal CT. Pol J Radiol. 2014;79:259-261.

5. Legmann P, Vignaux O, Dousset B. Rare and secondary tumors of the pancreas. In: Baert AL, editors. Radiology of the pancreas. 2nd ed. New York: Springer, Berlin Heidelberg; 1999. p. 295-310.

6. Butler JR, Fohtung TM, Sandrasegaran K, et al. The natural history of pancreatic lipoma: does it need observation. Pancreatology. 2016;16(1):95-98.

7. Kawahata S, Kawakami H, Kubota Y. A case of pancreatic lipoma with morphological change during long-term follow-up. Pancreas. 2017;46(8):e66-e67.

8. Minaga K, Yamashita Y, Uenoyama Y. Lipoma of the pancreas diagnosed by EUS-FNA. J Gastrointest Dig Syst. 2015;5:i105.

9. Matsumoto S, Mori $\mathrm{H}$, Miyake $\mathrm{H}$, et al. Uneven fatty replacement of the pancreas: Evaluation with CT. Radiology. 1995;194(2):453-458. 